\title{
Unraveling the repertoire in Wiskott-Aldrich syndrome
}

\author{
Sven H. Petersen ${ }^{1+}$, Anton Sendel ${ }^{1+}$, Mirjam van der Burg ${ }^{2}$ and Lisa S. Westerberg ${ }^{1 *}$ \\ ${ }^{1}$ Department of Microbiology Tumor and Cell Biology, Karolinska Institutet, Stockholm, Sweden \\ 2 Department of Immunology, Erasmus MC, University Medical Center Rotterdam, Rotterdam, Netherlands \\ ${ }^{*}$ Correspondence: lisa.westerberg@ki.se \\ ${ }^{\dagger}$ Sven H. Petersen and Anton Sendel have contributed equally to this work. \\ Edited by: \\ Luigi Daniele Notarangelo, Harvard Medical School, USA \\ Reviewed by: \\ Luigi Daniele Notarangelo, Harvard Medical School, USA \\ Anne Galy, Institut national de la santé et de la recherche médicale, France \\ Adrian Thrasher, University College London, UK
}

Keywords: Wiskott-Aldrich syndrome, WASp, B cell receptor, T cell receptor, immune repertoire diversity, next generation sequencing, spectratyping

Human immunology is entering the next frontier. With the fast developing technology, we can today sequence the whole genome from an individual in a relatively short time. It is now possible to decipher pathological mechanisms in immunological diseases, including primary immunodeficiencies, with high specificity. A particularly interesting aspect to study is the development and maintenance of the immune repertoire diversity and its consequences for disease progression. Until recently, a major difficulty in analysis of peripheral blood cells has been to sequence the locus encoding the $\mathrm{T}$ cell receptor (TCR) and the $\mathrm{B}$ cell receptor (BCR) in each cell. These receptors are assembled from a large array of $\mathrm{V},(\mathrm{D})$, and $\mathrm{J}$ gene segments in a process that inserts and deletes nucleotides in the $\mathrm{V}(\mathrm{D}) \mathrm{J}$ junctions (Figures 1A,B). The antigenic specificity of the BCR and TCR is determined by the complementaritydetermining regions (CDR) $1-3$, where the CDR3 covers the junctions between the $\mathrm{V}$, (D), and J segments and is the most variable part of the receptor (Figures 1A,B). To add to the complexity, $B$ cells undergo further gene diversification in the peripheral germinal centers by class switch recombination and induction of somatic hypermutations.

Mutations in the Wiskott-Aldrich syndrome protein (WASp) cause the severe immunodeficiency disease WiskottAldrich syndrome (WAS) $(1,2)$. WAS has been associated with numerous cellular defects and is termed a cell-trafficking disease of the immune system. WAS $^{-1-} \mathrm{B}$ cells are hyperactive and induce an autoreactive response, whereas $\mathrm{WAS}^{-1-} \mathrm{T}$ cells are hyporesponsive and $\mathrm{WAS}^{-l-} \mathrm{T}$ regulatory cells fail to suppress effector $\mathrm{T}$ cells $(1,2)$. Based on the important role of WASp for peripheral function, it has been somehow surprising that $\mathrm{B}$ and $\mathrm{T}$ cell development is intact as evident in normal progression through maturation stages in the bone marrow and thymus, respectively $(2,3)$. The role of WASp in creating a diverse BCR and TCR repertoire has until recently remained unknown. Since a skewed and oligoclonal BCR and TCR repertoire is linked to autoimmunity (2), a number of laboratories have now addressed the immune receptor repertoire in WAS patients. Two recent studies show that B cells from WAS patients have a decreased BCR repertoire, altered $V$ gene usage, and decreased somatic hypermutation $(4,5)$. In T cells, Wada et al. showed already in 2005 that the TCRV $\beta$ repertoire was skewed in WAS patients older than 15 years, while younger WAS patients showed no repertoire skewing (6). This year, Braun et al. and $\mathrm{Wu}$ et al. showed that also young WAS patients often had a skewed TCRV $\beta$ repertoire $(7,8)$.

O'Connell and colleagues (9) have in the present investigation examined the BCR and TCR repertoire in WAS patients using next generation sequencing [NGS, see recent reviews in Ref. $(10,11)]$. Using this technique, the authors collected a vast amount of data that allowed them to analyze the diversity of the receptor repertoire, including $\mathrm{V}(\mathrm{D}) \mathrm{J}$ segment usage, CDR3 size distribution, clonal expansions, and for BCRs; class switch recombination and frequency of somatic hypermutations. In B cells from WAS patients, the repertoire diversity tended to be lower than in controls, and the usage of some $\mathrm{V}$ heavy chain $(\mathrm{VH})$ gene segments was skewed. However, WAS B cells had normal rate of somatic hypermutation. In $\mathrm{T}$ cells of WAS patients, clonal expansions were present in the memory $\mathrm{CD} 4^{+}$ $\mathrm{T}$ cells and both in naive and memory $\mathrm{CD}^{+} \mathrm{T}$ cells. The usage of TCRV $\beta$ gene segments tended to be skewed to a higher extent in WAS patient $\mathrm{CD}^{+}$ $\mathrm{T}$ cells than in WAS patient $\mathrm{CD}^{+} \mathrm{T}$ cells. These results provide valuable information regarding the development and maintenance of the immune repertoire in WAS and importantly, describes alterations mainly in $\mathrm{CD} 8^{+} \mathrm{T}$ cells.

Interestingly, one out of the three patients in the study by O'Connell et al. showed increased VH4-34 expression that recognizes self-antigens (12). Two other recent studies have examined the BCR diversification in WAS patient $B$ cells and also found overrepresentation of the VH434 gene $(4,5)$. This suggests that autoreactive B cells are expanded in WAS patients, and the current study indicates that these B cells may be present even before clinical signs of autoimmune disease. Simon et al. and Castiello et al. describe decreased somatic hypermutation in WAS patients

Abbreviations: C, constant; CDR, complementary determining region; D, diversity; J, joining; N, non-templated; V, variable; WAS, Wiskott-Aldrich syndrome; WT, wildtype. 


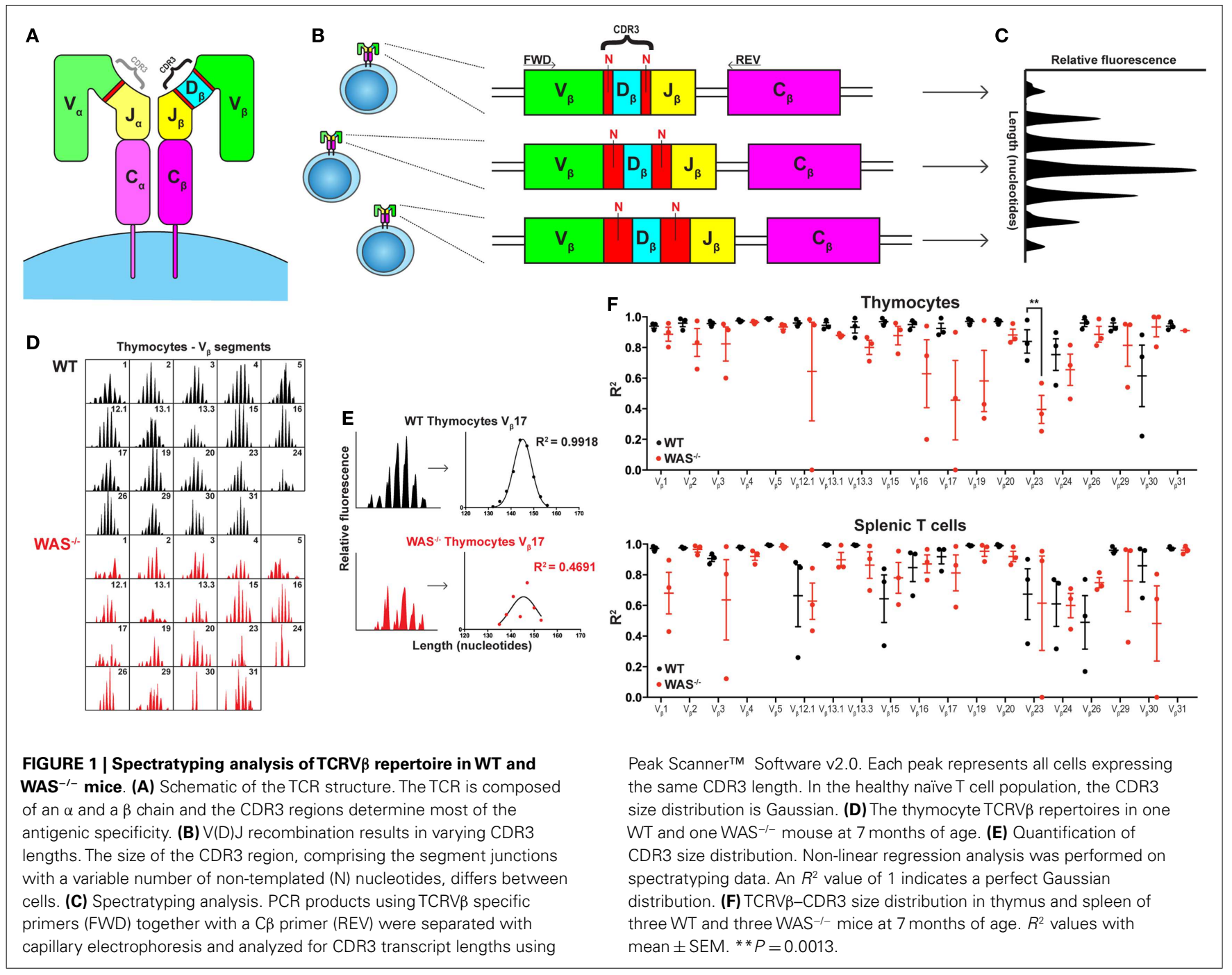

while the three patients in the O'Connell study showed normal somatic hypermutation. This may reflect differences in clinical status of the patients both in regards to infections and presence of autoimmune disease. In the $\mathrm{T}$ cell compartment, Wada et al. used the classical technique of CDR3-spectratyping (Figure 1C) and showed a skewed TCRV $\beta$ repertoire in WAS patients over 15 years of age, while younger patients showed a normal TCRV $\beta$ repertoire. These results suggested that thymic diversification may be intact. The authors reasoned that the oligoclonal TCR repertoire could reflect accumulation of $\mathrm{T}$ cell clones specific to autoantigens or result from chronic infections (6). However, using the same technique, Braun et al. recently showed that even young WAS patients often had a skewed TCRV $\beta$ repertoire (7). The work by O'Connell et al. extends these studies by usage of NGS on sorted $\mathrm{T}$ cell subsets and identifies restriction in the TCRV $\beta$ repertoire mainly in $\mathrm{CD}^{+} \mathrm{T}$ cells from young WAS patients. A recently published study supports this finding and shows that WAS mutations in young patients differentially influence the TCR diversity of different T cell subsets as determined by both CDR3-spectratyping and NGS. Compared with age-matched healthy control subjects, TCR diversity of WAS patient cells was severely skewed in memory/effector $\mathrm{CD}^{+} \mathrm{CD} 45 \mathrm{RO}^{+} \mathrm{T}$ cells and $\mathrm{CD}^{+}{ }^{+} \mathrm{CD} 45 \mathrm{RA}^{+} \mathrm{CCR}^{-}{ }^{-} \mathrm{T}_{\mathrm{EMRA}}$ cells (13).

A limitation of the CDR3-spectratyping (Figure 1C) is that unique sequences are not identified. As a result, it is not possible to distinguish if a peak in the spectrum represents many unique sequences of identical length or one clonally expanded TCR. The highly detailed data output obtained from NGS enables intricate analyses regarding the relationship between unique sequences and total sequences. O'Connell et al. show that WAS patients have reduced diversity of unique $\mathrm{BCR}$ and TCR sequences, indicating decreased capacity to diversify the receptor repertoire in the bone marrow and thymus, respectively. As the authors discuss, reduced diversity of unique sequences may also reflect decreased survival of $\mathrm{B}$ and $\mathrm{T}$ cells in the peripheral organs. Another explanation may be that NGS do not yet provide full sequence coverage and that underrepresented BCR and TCRs are excluded.

In all studies of WAS patients, it is difficult to fully define the cause for disturbed 
BCR and TCR repertoires. The repertoire may show alterations due to aberrant development, exposure to chronic infections (that may vary with age and in different countries), and/or autoimmune disease commonly seen in older WAS patients. In patients, addressing these interesting aspects would require analysis of newborns with a family history of disease. In an attempt to investigate age-related alterations in thymic output and to exclude potential burden of infections, we examined the TCRV $\beta$ repertoire in thymus and spleen of 4 weeks young and 7 months old WAS $^{-1-}$ mice housed in specific pathogenfree condition. In young $\mathrm{WAS}^{-1-}$ mice, the TCRV $\beta$ repertoire was indistinguishable to littermates controls and showed a Gaussian distribution reflecting the diverse TCR repertoire (unpublished data). In old mice, the TCRV $\beta$ repertoire of $\mathrm{WAS}^{-1-}$ mice showed more aberrations from the Gaussian distribution as compared to littermate control mice, both in thymus and spleen (Figures 1D-F). Our data suggest that in the absence of infections, autoantigens is the likely trigger of deviations from the diverse TCR repertoire in old mice. Moreover, the reduced TCRV $\beta$ repertoire diversity in thymocytes of old $\mathrm{WAS}^{-1-}$ mice support the notion raised by O'Connell et al. and Park et al. that thymic output is impaired in WAS (14).

Using NGS, valuable insights can be made in the study of the immune repertoire in human diseases such as WAS. The work by O'Connell and colleagues raises a number of intriguing questions, most importantly, how do relatively small changes in the BCR and TCR repertoire lead to severe disturbances in functionality of $\mathrm{B}$ and $\mathrm{T}$ cells? $\mathrm{WAS}^{-/-} \mathrm{B}$ cells are intrinsically hyperactive and have increased homeostatic expansion in the periphery (15). This together with an altered BCR repertoire as shown in the present study and in previous studies $(4,5)$, suggests that B cells drive autoimmune disease in WAS. O'Connell et al. show that memory $\mathrm{CD}^{+}$ $\mathrm{T}$ cells have a limited TCR repertoire and it has previously been shown that WAS $^{-1-}$ $\mathrm{CD} 4^{+} \mathrm{T}$ cells can drive autoimmune colitis (16). The present study raises the intriguing possibility that also $\mathrm{CD}^{+} \mathrm{T}$ cells may be autoreactive with a limited TCR repertoire as is often seen in diseases with disturbed thymopoiesis such as Omenn syndrome
(17). How this is related to previous findings of decreased cytotoxic capacity and reduced IFN $\gamma$ production by WAS $\mathrm{CD} 8^{+}$ $\mathrm{T}$ cells remains to be determined (18). Decreased production of IFN $\gamma$ is characteristic of $\mathrm{T}_{\mathrm{EMRA}}$ cells, a subset of $\mathrm{CD} 8^{+} \mathrm{T}$ cells that are terminally differentiated and functionally exhausted (19). T TMRA cells are expanded upon chronic viral infection, a common clinical complication in WAS patients. It is possible that the restricted TCR repertoire of $\mathrm{CD}^{+} \mathrm{T}$ cells in WAS patients to a large extent is represented by an expansion of $\mathrm{T}_{\mathrm{EMRA}}$ cells as shown in the present study by O'Connell et al. and in the recent paper by Wu et al. $(9,13)$.

What are the future challenges for NGS? An important question is to determine if peripheral blood lymphocytes represent $\mathrm{B}$ and $\mathrm{T}$ cells in tissues such as the spleen, lymph node, skin, and the gut. Peripheral blood B cells may represent as little as $2 \%$ of the body's total B cells (11). A recent study raises the intriguing possibility that B cell development occurs at sites in the intestinal mucosa and strikingly, that the gut BCR repertoire is vastly different from the repertoire in the bone marrow (20). This suggests that the commensal microbes influence antibody diversification in the gut and that this may be an important aspect to consider in analysis of peripheral blood from patients. NGS is superior to all previous approaches in the coverage of sequences. Nevertheless, the number of unique BCR and TCR sequences by far exceeds the number of sequences obtained by NGS (normally $10^{4}-10^{5}$ sequences as in the O'Connell study). Thus, with the current technology it may still prove difficult to obtain full coverage of the BCR and TCR repertoire. Another limitation is the reproducibility when analyzing rare sequences using alternative sequencing platforms. In one study, blood samples from the same donor were analyzed in parallel on the 454, Illumina, and Ion Torrent sequencing platforms (21). Profound discrepancies were revealed, with the three datasets differing significantly in terms of the diversity and relative abundance of clones within the overall repertoire.

This study identifies TCRs and BCRs that are clonally expanded in WAS patients. The next challenge is to identify the antigen recognized by a particular receptor. For B cells, Wardemann et al. described already in 2003 the method of single cell cloning and antibody expression to demonstrate that a large proportion of newly generated $B$ cells in fact express self- and poly-reactive BCRs (22). This method has been particularly useful in identification of self-antigens in autoimmune disease. Antigen recognition by TCRs is more complex since TCRs can only recognize antigenic peptides presented by MHC molecules by numerous unpredictable contacts (10). To identify the antigen recognition by a cloned TCR, the TCR can be screened over a library of MHC-peptides in insect cells or yeast cells. By comparing the identified antigenic peptide to candidate antigens in disease, the pathological peptide can be identified successfully (23).

In conclusion, the present study begins to unravel the BCR and TCR repertoire in WAS patients and paves the road for a better understanding of the differences in response to treatment such as bone marrow transplantation and gene therapy, the latter currently in clinical trial $(5,7,24)$.

\section{ACKNOWLEDGMENTS}

We would like to thank Dr. Anna Villa (San Raffaele Telethon Institute for Gene Therapy) for helpful comments. This work was supported by a MD PhD fellowship from Karolinska Institutet to Anton Sendel, a Vidi grant 91712323 from ZonMW/NOW to Mirjam van der Burg, and the Swedish Research Council, Cancer foundation, Childhood cancer foundation, the European Commission 7th framework program (Marie Curie \#249177), Jeansson Foundation, Groschinsky Foundation, Åke Wiberg Foundation, Bergvall Foundation, Swedish Society of Medicine, and Karolinska Institutet to Lisa S. Westerberg. Lisa S. Westerberg is a Ragnar Söderberg fellow in Medicine.

\section{REFERENCES}

1. Thrasher AJ, Burns SO. WASP: a key immunological multitasker. Nat Rev Immunol (2010) 10(3):182-92. doi:10.1038/nri2724

2. Catucci M, Castiello MC, Pala F, Bosticardo M, Villa A. Autoimmunity in Wiskott-Aldrich syndrome: an unsolved enigma. Front Immunol (2012) 3:209. doi:10.3389/fimmu.2012.00209

3. Thrasher AJ, Burns S. Wiskott-Aldrich syndrome: a disorder of haematopoietic cytoskeletal regulation. Microsc Res Tech (1999) 47(2):107-13. doi:10.1002/(SICI)1097-0029(19991015)47: 2<107::AID-JEMT3>3.0.CO;2-H 
4. Simon KL, Anderson SM, Garabedian EK, Moratto D, Sokolic RA, Candotti F. Molecular and phenotypic abnormalities of B lymphocytes in patients with Wiskott-Aldrich syndrome. J Allergy Clin Immunol (2014) 133(3):896e-9e. doi:10.1016/j. jaci.2013.08.050

5. Castiello MC, Bosticardo M, Pala F, Catucci M, Chamberlain N, van Zelm MC, et al. WiskottAldrich syndrome protein deficiency perturbs the homeostasis of B-cell compartment in humans. J Autoimmun (2014) 50:42-50. doi:10.1016/j.jaut. 2013.10.006

6. Wada T, Schurman SH, Garabedian EK, Yachie A, Candotti F. Analysis of T-cell repertoire diversity in Wiskott-Aldrich syndrome. Blood (2005) 106(12):3895-7. doi:10.1182/blood-200506-2336

7. Braun CJ, Boztug K, Paruzynski A, Witzel M, Schwarzer A, Rothe $M$, et al. Gene therapy for Wiskott-Aldrich syndrome - longterm efficacy and genotoxicity. Sci Transl Med (2014) 6(227):227ra33. doi:10.1126/scitranslmed. 3007280

8. Wu X, Yoo Y, Okuhama NN, Tucker PW, Liu G, Guan JL. Regulation of RNA-polymeraseII-dependent transcription by N-WASP and its nuclear-binding partners. Nat Cell Biol (2006) 8(7):756-63. doi:10.1038/ncb1433

9. O’Connell AE, Volpi S, Dobbs K, Fiorini C, Tsitsikov E, de Boer $\mathrm{H}$, et al. Next generation sequencing reveals skewing of the $\mathrm{T}$ and $\mathrm{B}$ cell receptor repertoires in patients with Wiskott-Aldrich syndrome. Front Immunol (2014) 5:340. doi:10.3389/ fimmu.2014.00340

10. Newell EW, Davis MM. Beyond model antigens: high-dimensional methods for the analysis of antigen-specific T cells. Nat Biotechnol (2014) 32(2):149-57. doi:10.1038/nbt.2783

11. Georgiou G, Ippolito GC, Beausang J, Busse CE, Wardemann H, Quake SR. The promise and challenge of high-throughput sequencing of the antibody repertoire. Nat Biotechnol (2014) 32(2):158-68. doi:10.1038/nbt.2782

12. Dorner T, Lipsky PE. Molecular basis of immunoglobulin variable region gene usage in systemic autoimmunity. Clin Exp Med (2005) 4(4):159-69. doi:10.1007/s10238-004-0051-2

13. Wu J, Liu D, Tu W, Song W, Zhao X. T-cell receptor diversity is selectively skewed in T-cell populations of patients with Wiskott-Aldrich syndrome. J Allergy Clin Immunol (2014). doi:10.1016/j.jaci. 2014.06.025

14. Park JY, Kob M, Prodeus AP, Rosen FS, Shcherbina A, Remold-O’Donnell E. Early deficit of lymphocytes in Wiskott-Aldrich syndrome: possible role of WASP in human lymphocyte maturation. Clin Exp Immunol (2004) 136(1):104-10. doi:10.1111/ j.1365-2249.2004.02409.x

15. Recher M, Burns SO, de la Fuente MA, Volpi S, Dahlberg C, Walter JE, et al. B cellintrinsic deficiency of the Wiskott-Aldrich syndrome protein (WASp) causes severe abnormalities of the peripheral B-cell compartment in mice. Blood (2012) 119(12):2819-28. doi:10.1182/ blood-2011-09-379412

16. Maillard MH, Cotta-de-Almeida V, Takeshima F, Nguyen DD, Michetti P, Nagler C, et al. The Wiskott-Aldrich syndrome protein is required for the function of CD4(+)CD25(+)Foxp3(+) regulatory T cells. J Exp Med (2007) 204(2):381-91. doi:10.1084/jem.20061338

17. van der Burg M, van Zelm MC. Clinical spectrum of SCID: the key is in the thymus? Front Immunol (2014) 5:111. doi:10.3389/fimmu.2014.00111

18. Massaad MJ, Ramesh N, Geha RS. WiskottAldrich syndrome: a comprehensive review. Ann N Y Acad Sci (2013) 1285:26-43. doi:10.1111/nyas. 12049

19. Kaech SM, Cui W. Transcriptional control of effector and memory CD8+ T cell differentiation. Nat Rev Immunol (2012) 12(11):749-61. doi:10.1038/ nri3307

20. Wesemann DR, Magee JM, Boboila C, Calado DP, Gallagher MP, Portuguese AJ, et al. Immature B cells preferentially switch to $\operatorname{IgE}$ with increased direct Smu to Sepsilon recombination. J Exp Med (2011) 208(13):2733-46. doi:10.1084/ jem.20111155

21. Bolotin DA, Mamedov IZ, Britanova OV, Zvyagin IV, Shagin D, Ustyugova SV, et al. Next generation sequencing for TCR repertoire profiling: platformspecific features and correction algorithms. Eur J Immunol (2012) 42(11):3073-83. doi:10.1002/eji. 201242517

22. Wardemann H, Yurasov S, Schaefer A, Young JW, Meffre E, Nussenzweig MC. Predominant autoantibody production by early human B cell precursors. Science (2003) 301(5638):1374-7. doi:10. $1126 /$ science. 1086907

23. Su LF, Kidd BA, Han A, Kotzin JJ, Davis MM. Virus-specific CD4(+) memory-phenotype T cells are abundant in unexposed adults. Immunity (2013) 38(2):373-83. doi:10.1016/j.immuni.2012. 10.021

24. Aiuti A, Biasco L, Scaramuzza S, Ferrua F, Cicalese MP, Baricordi C, et al. Lentiviral hematopoietic stem cell gene therapy in patients with Wiskott-Aldrich syndrome. Science (2013) 341(6148):1233151. doi:10.1126/science. 1233151

Conflict of Interest Statement: The authors declare that the research was conducted in the absence of any commercial or financial relationships that could be construed as a potential conflict of interest.

Received: 12 August 2014; accepted: 11 October 2014; published online: 27 October 2014

Citation: Petersen SH, Sendel A, van der Burg M and Westerberg LS (2014) Unraveling the repertoire in Wiskott-Aldrich syndrome. Front. Immunol. 5:539. doi: 10.3389/fimmu.2014.00539

This article was submitted to Primary Immunodeficiencies, a section of the journal Frontiers in Immunology. Copyright (C) 2014 Petersen, Sendel, van der Burg and Westerberg. This is an open-access article distributed under the terms of the Creative Commons Attribution License (CC BY). The use, distribution or reproduction in other forums is permitted, provided the original author(s) or licensor are credited and that the original publication in this journal is cited, in accordance with accepted academic practice. No use, distribution or reproduction is permitted which does not comply with these terms. 